\title{
Myxedema coma precipitated by diabetic ketoacidosis after total thyroidectomy: a case report
}

\author{
Jin Joo Kim and Eun Young Kim ${ }^{*}$
}

\begin{abstract}
Background: Myxedema coma is profound decompensated hypothyroidism usually precipitated by stressors, and its occurrence in association with total thyroidectomy or metabolic disorders, such as diabetic ketoacidosis, is unusual.

Case presentation: A 43-year-old Asian man with history of total thyroidectomy who was scheduled for a second radioactive iodine therapy presented to our hospital with decreased mental status and hyperglycemia. He had a history of thyroid cancer but did not have diabetes mellitus. He was in a hypothermic state and had a Glasgow Coma Scale score of 10 out of 15 at presentation; arterial blood gas analysis revealed a state of metabolic acidosis and laboratory findings suggested hyperglycemia with glycosuria, ketoacidosis, and severe hypothyroidism. A thyroid function test showed thyroid-stimulating hormone of $34.126 \mathrm{ulU} / \mathrm{mL}$, free thyroxine of $1.02 \mathrm{ng} / \mathrm{dL}$, and triiodothyronine of $1.04 \mathrm{ng} / \mathrm{mL}$. The glycated hemoglobin of this patient was checked due to hyperglycemia and the value of glycated hemoglobin was $16.5 \%$ which met the criteria for a diagnosis of diabetes. After treatment for myxedema with liothyronine 5 mcg two times per day and levothyroxine 175 mcg once daily via a nasogastric tube and diabetic ketoacidosis with intravenously administered fluid and insulin, his clinical condition rapidly improved including mental status, hyperglycemia, and acidosis. During the hospitalization, a workup for diabetes mellitus was performed and the results suggested that a diagnosis of type 2 diabetes mellitus would be appropriate.

Conclusions: This case demonstrated that diabetic ketoacidosis not only could be a potential contributor to myxedema coma but also mask typical clinical features, making diagnosis more difficult. Considering the possibility of an increasing number of potential patients with hypothyroidism developed after thyroidectomy, constant vigilance is required for a better clinical outcome, including early recognition and management in critical care in advance for unusual diabetic ketoacidosis which could precipitate decompensated hypothyroidism.
\end{abstract}

Keywords: Myxedema coma, Diabetic ketoacidosis, Total thyroidectomy, Hypothyroidism

\section{Background}

Myxedema coma is one of the potentially lethal complications of hypothyroidism, and the term might account for profound decompensated hypothyroidism caused by a stressor such as infection. Although myxedema coma is rare, the mortality rate has been reported to be high: approximately $50-60 \%$ in recent studies $[1,2]$. Early recognition and more aggressive management have been

\footnotetext{
*Correspondence: freesshs@naver.com

Division of Trauma and Surgical Critical Care, Department of Surgery, Seoul St. Mary's Hospital, College of Medicine, The Catholic University of Korea, Banpo-daero 222, Seocho-gu, Seoul 137-701, Korea
}

noted to be important because they are expected to reduce the mortality rate to closer to $20-25 \%$ [3].

Myxedema coma usually seems to be more common in older women during the winter period; it is also known to be precipitated by environmental cold exposure, infection, sepsis, stroke, cardiovascular compromise, or medication. There are several clinical features including hypothermia, altered mental status, bradycardia, hypotension, hypercapnia, or delayed deep tendon reflexes. An electrocardiogram shows QT prolongation and sinus bradycardia in most cases, and gastric atony or paralytic ileus can be seen from plain X-ray. The

$$
\begin{aligned}
& \text { (c) The Author(s). } 2019 \text { Open Access This article is distributed under the terms of the Creative Commons Attribution } 4.0 \\
& \text { International License (http://creativecommons.org/licenses/by/4.0/), which permits unrestricted use, distribution, and } \\
& \text { reproduction in any medium, provided you give appropriate credit to the original author(s) and the source, provide a link to } \\
& \text { the Creative Commons license, and indicate if changes were made. The Creative Commons Public Domain Dedication waiver } \\
& \text { (http://creativecommons.org/publicdomain/zero/1.0/) applies to the data made available in this article, unless otherwise stated. }
\end{aligned}
$$


diagnosis of myxedema coma can be made with a patient who has a history of thyroid disease or thyroidectomy with altered mental status or hypothermia. However, it can be misdiagnosed due to various clinical conditions that can present several manifestations similar to those of myxedema coma, such as sepsis, heart failure, cerebrovascular disease, drug use, or metabolic disorder [4]. Here we report the case of a 43-year-old man with undiagnosed diabetes mellitus who showed atypical clinical presentation of myxedema coma precipitated by diabetic ketoacidosis (DKA). Study approvals were obtained from the Institutional Review Board (IRB) of Seoul St. Mary's Hospital (No. IRB; KC18ZESI0785).

\section{Case presentation}

A 43-year-old Asian man presented to the emergency department in our institution due to generalized weakness in April 2018. One month prior to admission, his family noted that he showed poor oral intake and consistently complained of epigastric discomfort. He was diagnosed as having impaired fasting glucose and hyperlipidemia at the age of 42 on routine medical checkup. Eight months ago, he underwent total thyroidectomy with both central and sentinel lymph node dissection due to papillary thyroid carcinoma and the pathologic stage was diagnosed as T3N1bM0 on the permanent pathologic report. After that, the first radioactive iodine (RAI) therapy was conducted and an iodine [1-3] whole body scan was planned to determine whether to perform the second RAI that was on the next day of visiting the emergency room, therefore, he had to stop the thyroid medication for 3 weeks to prepare for the examination.

At the time of admission to the emergency room, he was noted to be somnolent and had a decreased level of consciousness. He opened eyes to pain, showed inappropriate verbal response and flexion withdrawal from pain, which suggested that Glasgow Coma Scale (GCS) was 10 out of 15 . On physical examination, there was no pretibial edema and his pupils were equal in size and normally reactive to light. His abdomen was slightly distended with decreased bowel sound and his extremities were cold. His blood pressure was 127/96 mmHg, heart rate was 101 beats per a minute, and respiratory rate was 25 breaths per a minute with oxygen saturation $97 \%$ on room air. He was in a hypothermic state and his tympanic temperature was approximately $34.0{ }^{\circ} \mathrm{C}$. Chest radiography revealed the findings of gastroparesis and paralytic ileus as presented in Fig. 1. An electrocardiogram at presentation showed sinus tachycardia with QT prolongation by $537 \mathrm{~ms}$ of corrected QT interval (Fig. 2).

Arterial blood gas analysis revealed a state of metabolic acidosis: a pH of 7.00, partial pressure of carbon dioxide in arterial blood $\left(\mathrm{PaCO}_{2}\right)$ of less than $10 \mathrm{mmHg}$,

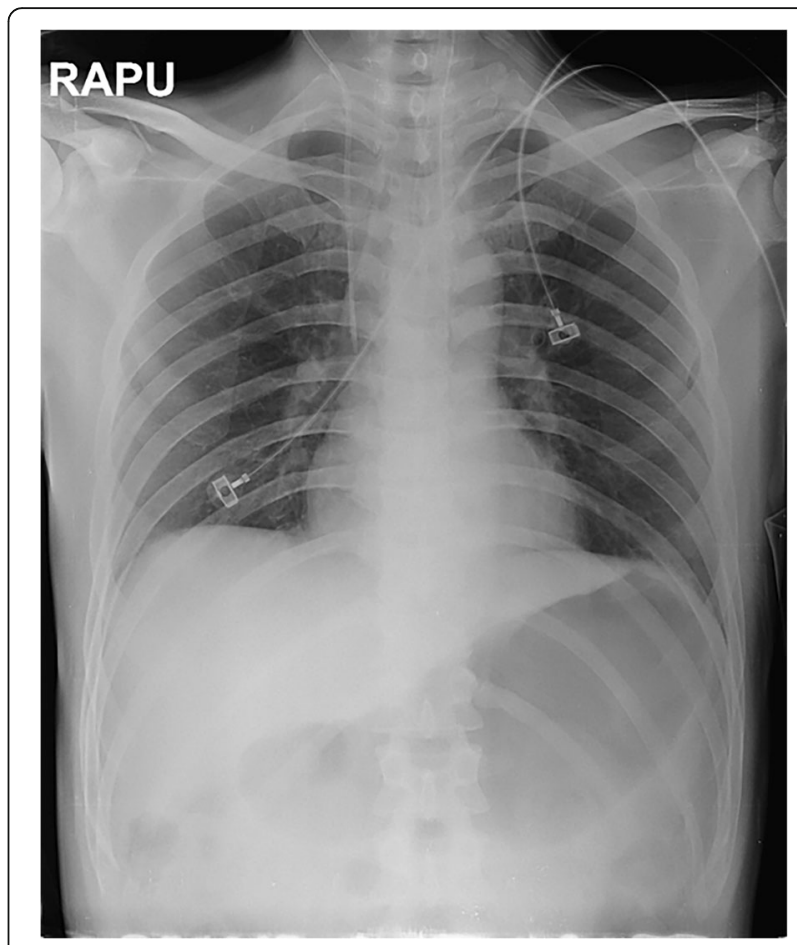

Fig. 1 Chest X-ray at presentation

bicarbonate $\left(\mathrm{HCO}_{3}\right)$ of less than $10 \mathrm{mmol} / \mathrm{L}$, and base excess of - 34.6. Laboratory findings suggested hyperglycemia with glycosuria and ketoacidosis, which are presented in Table 1. Considering the history of total thyroidectomy and planned schedule for RAI, a thyroid function test (TFT) was conducted and revealed severe hypothyroidism. He was found to have a thyroidstimulating hormone $(\mathrm{TSH})$ of $34.126 \mathrm{uIU} / \mathrm{mL}(0.55-$ $4.78 \mathrm{uIU} / \mathrm{mL}$ ) and free thyroxine (T4) of less than 0.01 $\mathrm{ng} / \mathrm{dL}(0.82-1.76 \mathrm{ng} / \mathrm{dL})$ and triiodothyronine (T3) of less than $0.01 \mathrm{ng} / \mathrm{mL}(0.6-1.81 \mathrm{ng} / \mathrm{mL})$. Even though he did not have any history of diabetes mellitus, we checked his glycated hemoglobin (HbA1c) due to hyperglycemia. Finally, the value of HbA1c was $16.5 \%$ which met the criteria for a diagnosis of diabetes.

He was admitted to the intensive care unit (ICU) for the management of DKA and myxedema coma. He received intravenously administered fluid with electrolytes and an immediately applied insulin pump. For hormonal replacement, liothyronine $5 \mathrm{mcg}$ two times per day and levothyroxine $175 \mathrm{mcg}$ once daily were administered via a nasogastric tube. He instantly responded to the therapy with a favorable clinical improvement. His mental status started to improve several hours after treatment and at the third day of hospitalization he showed a GCS of $15 / 15$; his body temperature increased from $34{ }^{\circ} \mathrm{C}$ to $36.5^{\circ} \mathrm{C}$ approximately 10 hours after admission. The metabolic acidosis was corrected 6 hours after administration of intravenously administered fluid with insulin 


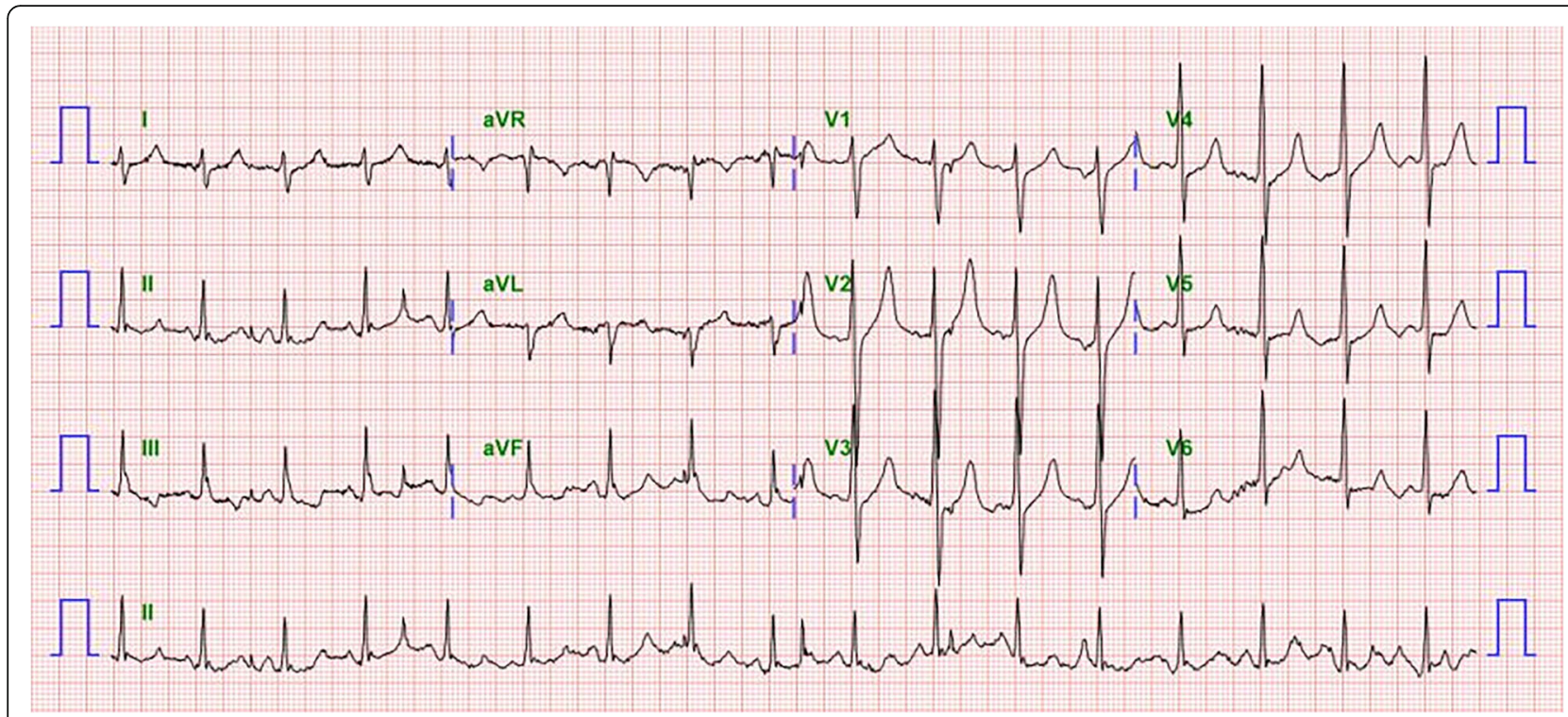

Fig. 2 Electrocardiogram at presentation

pump and hyperglycemia was also improved; the insulin pump was discontinued then and switched to subcutaneous insulin 1 day after hospitalization. Repeated TFT before discharge revealed TSH of $21.798 \mathrm{uIU} / \mathrm{mL}(0.55-$ $4.78 \mathrm{uIU} / \mathrm{mL})$, free $\mathrm{T} 4$ of $1.02 \mathrm{ng} / \mathrm{dL}(0.82-1.76 \mathrm{ng} / \mathrm{dL})$, and $\mathrm{T} 3$ of $1.04 \mathrm{ng} / \mathrm{dL}(0.6-1.81 \mathrm{ng} / \mathrm{mL})$. The clinical course of this patient was summarized in Table 2. During the hospitalization, a workup for diabetes mellitus was performed and there was no evidence of pancreas mass or pancreatitis on abdominal computed tomography (Fig. 3). Results from investigations for diabetes mellitus showed a fasting c-peptide of $1.08 \mathrm{ng} / \mathrm{mL}(0.48-$ $3.30 \mathrm{ng} / \mathrm{mL}$ ), anti-islet cell antibodies (ab) negative, and glutamic acid decarboxylase (GAD) ab of $0.01 \mathrm{U} / \mathrm{ml}$

Table 1 Laboratory investigations at admission to the Intensive care Unit

\begin{tabular}{lll}
\hline & Values & Reference range \\
\hline WBC $\left(\times 10^{9} / \mathrm{L}\right)$ & 25.97 & $4.0-10.0$ \\
$\mathrm{Hb}(\mathrm{g} / \mathrm{dl})$ & 16.8 & $13.0-18.0$ \\
$\mathrm{PLT}(\times 103 / \mathrm{L})$ & 395 & $130-400$ \\
Serum glucose $(\mathrm{mg} / \mathrm{dl})$ & 1020 & $50-100$ \\
Urine glucose & 3 positive & Negative \\
Creatinine $(\mathrm{mg} / \mathrm{dl})$ & 2.68 & $0.6-1.2$ \\
Serum osmolarity $(\mathrm{mosm} / \mathrm{kg})$ & 386 & $28-120$ \\
Serum sodium $(\mathrm{mEq} / \mathrm{L})$ & 138 & $136-146$ \\
Serum potassium $(\mathrm{mEq} / \mathrm{L})$ & 5.9 & $3.5-5.1$ \\
Serum chloride $(\mathrm{mEq} / \mathrm{L})$ & 84 & $98-106$ \\
Serum total ketone bodies $(\mathrm{umol} / \mathrm{L})$ & $>1000$ & $20-120$ \\
Urine ketone & 3 positive & Negative \\
\hline
\end{tabular}

Hb hemoglobin, PLT platelets, WBC white blood cells which suggested that a diagnosis of type 2 diabetes mellitus would be appropriate.

He was discharged from surgical ICU after 2 days, stayed for a further 8 days on the general ward and was discharged on the 11th hospital day with tolerable status. The dose of thyroid hormone medications was subsequently reduced at our out-patient clinic after he was discharged and an endocrinologist recommend insulin with orally administered hypoglycemic agents.

\section{Discussion and conclusions}

Myxedema coma is a medical status in which there is a complex manifestation of decompensated hypothyroidism with hypothermia, hypotension, or decreased level of consciousness. It is known to be a true medical emergency; although it is rare, the overall mortality was reported to be $50-60 \%$ in the past. Most cases occur in older women during winter periods; it can be associated with signs of hypothyroidism. A high degree of suspicion is necessary for diagnosis in patients presenting with altered level of consciousness who have a history of thyroidectomy, hypothyroidism, RAI therapy, or a discontinuation or improper administration of thyroid medications because early recognition and intensive supportive care in advance have reduced the mortality rate to $20-25 \%$ [2, 3]. Kumar et al. [5] previously reported rapid clinical improvement of hemodynamics and respiratory status after thyroid hormone administration in patients with hypothyroid state superimposed on critical illness who did not have underlying thyroid disease. However, careful individual assessment would be needed considering all possible clinical conditions to interpret the result of TFT for critically ill patients. And, 
Table 2 Clinical course during hospitalization

\begin{tabular}{|c|c|c|c|c|c|c|c|c|c|c|c|}
\hline & $\begin{array}{l}\text { Glucose } \\
\mathrm{mg} / \mathrm{L}\end{array}$ & $\begin{array}{l}\mathrm{AG} \\
\mathrm{nmol} / \mathrm{L}\end{array}$ & $\begin{array}{l}\mathrm{Na} \\
\mathrm{mEq} / \mathrm{L}\end{array}$ & $\begin{array}{l}\mathrm{K} \\
\mathrm{mEq} / \mathrm{L}\end{array}$ & $\begin{array}{l}\mathrm{Cl} \\
\mathrm{mEq} / \mathrm{L}\end{array}$ & $\begin{array}{l}\text { Osm } \\
\text { mosm/L }\end{array}$ & $\begin{array}{l}\text { TSH ull/ } \\
\mathrm{mL}\end{array}$ & $\begin{array}{l}\text { Free T4 ng/ } \\
\text { dL }\end{array}$ & $\begin{array}{l}\text { T3 ng/ } \\
\mathrm{dL}\end{array}$ & $\begin{array}{l}\text { Neurologic } \\
\text { status }\end{array}$ & \\
\hline Admission & 1020 & $>44$ & 138 & 5.9 & 84 & 386 & 34.126 & $<0.01$ & $<0.01$ & GCS 10/15 & $\begin{array}{l}\text { Insulin pump } \\
\text { IVF } \\
\text { Liothyronine } \\
\text { Levothyroxine }\end{array}$ \\
\hline HD \#2 & 306 & 19.5 & 149 & 4.0 & 110 & 331 & & & & GCS 14/15 & \\
\hline HD \#3 & 278 & 11.45 & 143 & 3.6 & 108 & 308 & 41.399 & 0.19 & $<0.01$ & GCS 15/15 & Insulin SQ \\
\hline $\begin{array}{l}\text { Discharge (HD } \\
\# 11)\end{array}$ & 145 & & 138 & 4.5 & 101 & 296 & 21.798 & 1.02 & 1.04 & & \\
\hline
\end{tabular}

AG anion gap, GCS Glasgow Coma Scale, HD hospital day, IVF intravenously administered fluid, Osm calculated serum osmolarity, SQ subcutaneous, T3 triiodothyronine, $T 4$ thyroxine, $T S H$ thyroid-stimulating hormone

regarding causation of our case, we believe that myxedema coma could be a more suitable conclusion.

Previous studies have suggested several poor prognostic factors for myxedema coma and Jordan [6] suggested that old age, bradycardia less than 44 beats per minute, a low serum level of T3, hypothermia of less than $34^{\circ} \mathrm{C}$, and persistent hypotension are risk factors for poor prognosis of myxedema coma. Sepsis and myocardial infarction, and delayed treatment could also affect a poor prognosis. Rodriguez et al. [3] also observed that an Acute Physiology and Chronic Health Evaluation (APACHE) II score of more than 20 and the degree of consciousness could determine the prognosis of myxedema coma and it could be precipitated by infection, cardiac events, medications such as antiepileptic drugs, trauma, surgical stress, or exposure to cold temperature. However, few cases have been reported in which myxedema coma could be precipitated by metabolic disorders such as DKA or hyperglycemic hyperosmolar state (HHS) which could also show hyperglycemia, altered mental status, or hypotension [1, 7]. In this patient, DKA would have led to rapid development of myxedema coma albeit he had not been diagnosed as having diabetes mellitus. We believe that DKA could act as a major contributor

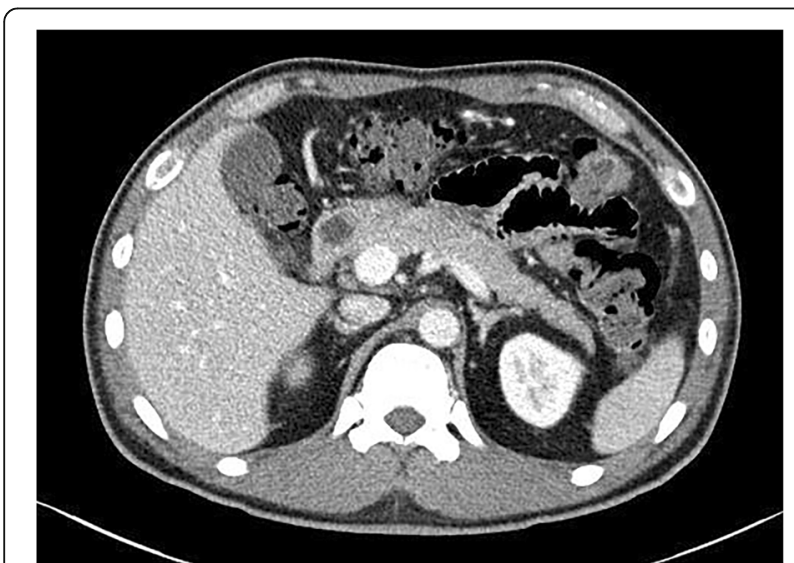

Fig. 3 Computed tomography of pancreas. There was no evidence of pancreas mass or pancreatitis to the development of myxedema coma, as our patient did not have a period of a long-standing hypothyroidism. In this case, the patient did not have other significant risk factors for myxedema coma such as myocardial infarction or history of medication use except thyroid hormone at presentation.

To the best of our knowledge, there are no reports in the English literature about a case of myxedema coma developing after withdrawal of hormone replacement therapy among patients after thyroidectomy for thyroid cancer or those who are scheduled for RAI therapy. In addition, myxedema coma precipitated by DKA has been described in only two cases in the English literature: one case was precipitated by DKA with neuroleptic drugs, in the other case it was believed that myxedema coma was precipitated by DKA only. However, from both cases, the patients did not have a history of surgical resection of thyroid due to thyroid cancer $[4,8]$. Our case is an unusual case of myxedema coma because of the middle age of our patient, absence of history of cold exposure, and having atypical clinical features. It would be unlikely that withdrawal of $40 \mathrm{mg}$ daily of liothyronine for a few weeks could precipitate a myxedema coma, even with an associated DKA [8]. The hallmark of myxedema is alteration of mental status such as lethargy or coma and hypothermia. The hypothermia could be the clinical clue for diagnosis. This patient showed hypothermia with decreased GCS; however, our patient also had atypical features of myxedema such as normal respiratory rate, normal heart rate, and normal blood pressure.

Myxedema would also be typically associated with hyponatremia, hypo-osmolality, and hypoglycemia because of increased antidiuretic hormone production as a result of decreased cardiac output, and reduced renal plasma flow, which could increase total body water [7]. In our case, plasma osmolality was increased with normonatremia which were fairly mild for DKA. It could be possible that coexistence with myxedema may improve the hyperosmolar state of DKA. Although our patient had not been diagnosed previously for diabetes mellitus, he had marked hyperglycemia with high anion gap 
metabolic acidosis, and significant blood and urine ketones which implied DKA. Moreover, a diagnostic workup for hyperglycemia suggested that a diagnosis of type 2 diabetes mellitus would be appropriate. Actually, DKA was usually regarded to occur only in patients with type 1 diabetes mellitus, however, a retrospective study recently found that among adult patients with DKA, $47 \%$ were classified as type 1 diabetes, $26 \%$ had known type 2 diabetes, and $27 \%$ had DKA as the initial presentation of the diabetes [9]. We suppose that this point would be an impressive clinical manifestation of this patient compared with patients who have an ordinary case of DKA.

The interesting viewpoint of our case includes unusual clinical manifestations, the absence of long-standing hypothyroidism, no history of cold exposure, and the masking of typical clinical features by DKA. We suppose that the diabetes of the patient, who was healthy despite having risk factors for diabetes, was expressed in the form DKA which was triggered by transient severe decompensated hypothyroidism during the RAI process. The incidence of thyroid cancer has increased worldwide and the treatment of thyroid cancer is thyroidectomy followed by RAI [10]. In 2012, approximately two thirds of patients with thyroid cancer underwent radical thyroidectomy and approximately $60 \%$ of them received RAI in South Korea $[9,11]$. In addition, the prevalence of diabetes mellitus has increased in South Korea and it has been shown that the increased incidence of the lifestyle-related disease is associated with obesity or decreased physical activity, which is a result of urbanization or a change of diet as the undiagnosed risk factors [12]. Therefore, we expect that, similar to this case, the risk of development of a medically critical illness such as myxedema coma precipitated by DKA will be relatively high in patients with unrecognized diabetes and consideration of this clinical condition will be needed.

In conclusion, early recognition of atypical myxedema coma, management in critical care in advance, and timely administration of thyroid hormone therapy are important for better clinical outcome of myxedema coma. Our case demonstrated that DKA not only could be a potential contributor to myxedema coma but also mask typical clinical features, making diagnosis more difficult. Considering the possibility of an increasing number of potential patients with hypothyroidism developed after thyroidectomy, constant vigilance is necessary for unusual DKA which could precipitate decompensated hypothyroidism.

\section{Abbreviations}

Ab: Antibodies; APACHE: Acute Physiology and Chronic Health Evaluation; DKA: Diabetic ketoacidosis; DM: Diabetes mellitus; GAD: Glutamic acid decarboxylase; GCS: Glasgow Coma Scale; HbA1c: Glycated hemoglobin; $\mathrm{HCO}_{3}$ : Bicarbonate; HHS: Hyperglycemic hyperosmolar state; ICU: Intensive care unit; IRB: Institutional Review Board; $\mathrm{PaCO}_{2}$ : Partial pressure of carbon dioxide in arterial blood; RAl: Radioactive iodine; T3: Triiodothyronine; T4: Thyroxine; TFT: Thyroid function test; TSH: Thyroid-stimulating hormone

Acknowledgements

Not applicable.

Funding

None declared.

Availability of data and materials

Availability of data and materials can be acquired through contact with the corresponding author.

\section{Authors' contributions}

Both JJ and EY contributed equally to the design, drafting, and editing of this manuscript. Both authors read and approved the final manuscript.

Ethics approval and consent to participate

Study approvals were obtained from the IRB of Seoul St. Mary's Hospital (No. IRB; KC18ZESI0785). Informed consent was obtained from the patient.

\section{Consent for publication}

Written and informed consent was obtained from the patient for publication of the case report and the accompanying images. Copies of the written consent forms are available for review by the Editor-in-Chief of this journal.

\section{Competing interests}

The authors declare that they have no competing interests.

\section{Publisher's Note}

Springer Nature remains neutral with regard to jurisdictional claims in published maps and institutional affiliations.

Received: 5 December 2018 Accepted: 22 January 2019

Published online: 04 March 2019

\section{References}

1. Tews MC, Shah SM, Gossain W. Hypothyroidism: mimicker of common complaints. Emerg Med Clin North Am. 2005;23(3):649-67. vii

2. Wartofsky L. Myxedema coma. Endocrinol Metab Clin N Am. 2006;35(4):68798. vii-viii

3. Rodriguez I, Fluiters E, Perez-Mendez LF, Luna R, Paramo C, Garcia-Mayor RV. Factors associated with mortality of patients with myxoedema coma: prospective study in 11 cases treated in a single institution. J Endocrinol. 2004;180:347-50.

4. Kelekar G, Milanesi A, Yu R. Myxedema Coma Precipitated by Diabetic Ketoacidosis: An Atypical Case. ICU Dir. 2010;1(2):66-70.

5. Kumar E, McCurdy MT, Koch CA, Hamadah A, Fulop T, Gharaibeh KA. Impairment of Thyroid Function in Critically III Patients in the Intensive Care Units. Am J Med Sci. 2018;355:281-5.

6. Jordan RM. Myxedema coma: Pathophysiology, Therapy, and Factors Affecting Prognosis. Med Clin N Am. 1995;79(1):185-94.

7. Spyridoulias A, Riaz MS. Myxoedema coma in the setting of hyperglycaemic hyperosmolar state. BMJ Case Rep. 2016;2016 https://doi.org/10.1136/ bcr-2015-213411.

8. Cappelli C, Stanga B, Paini A, Gandossi E, Cumetti D, Castellano M, et al. Myxoedema coma precipitated by diabetic ketoacidosis and neuroleptic drugs: case report in an intensive care unit. Intern Emerg Med. 2007;2(2):147-9.

9. Ahn HS, Kim HJ, Welch HG. Korea's Thyroid-Cancer "Epidemic" — Screening and Overdiagnosis. N Engl J Med. 2014;371(19):1765-7.

10. Kim TY, Kim WG, Kim WB, Shong YK. Current Status and Future Perspectives in Differentiated Thyroid Cancer. Endocrinol Metab. 2014; 29(3):217-25.

11. Song YD, Park KH, Yook TM, Nam J. Trends of thyroid cancer over the past decade; National Health Insurance Service claim database: National Health Insurance Service Ilsan Hospital; 2014. http://lib.nhis.or.kr/search/ detail/CAT000000016611

12. Hwangbo $Y$, Kang $D$, Kang $M$, et al. Incidence of diabetes after cancer development: A korean national cohort study. JAMA Oncol. 2018:4(8): 1099-105. 